\title{
Trophoblast Cell Surface Antigen 2 (Trop-2) Protein is Highly Expressed in Salivary Gland Carcinomas and Represents a Potential Therapeutic Target
}

\author{
Philipp Wolber $^{1}$ [D - Lisa Nachtsheim ${ }^{1} \cdot$ Franziska Hoffmann $^{2,3}$. Jens Peter Klußmann ${ }^{1,4} \cdot$ Moritz Meyer $^{5}$.

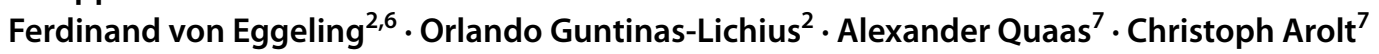

Received: 23 November 2020 / Accepted: 10 February 2021 / Published online: 22 April 2021

(c) The Author(s) 2021

\begin{abstract}
Treatment options for unresectable, recurrent or metastatic salivary gland carcinomas (SGC) are scarce. Trophoblast cell surface antigen 2 (Trop-2) is a transmembrane glycoprotein that is involved in a variety of oncogenic cell signaling pathways. Its potential as a target for the antibody-drug conjugate sacituzumab govitecan has already been demonstrated in different tumor entities. The United States Food and Drug Administration approved this antibody-drug conjugate for the treatment of metastatic triple-negative breast cancer. Here, we aimed to investigate Trop-2 protein expression in different entities of SGCs. We retrospectively reviewed the medical records of all patients that underwent surgery for a primary SGC in a tertiary referral center between 1990 and 2014. Immunohistochemical (IHC) staining for Trop-2 was performed and rated as negative, weak, moderate or high using a semiquantitative score. Additionally, representative cases were analyzed using MALDI-mass spectrometry (MS) imaging to confirm the IHC results. The cohort consisted of 114 tumors of the parotid gland (90.4\%) and submandibular gland (9.6\%). It mainly included mucoepidermoid, salivary duct and adenoid cystic carcinomas. In IHC samples, $44 \%$ showed high, $38 \%$ moderate and $10 \%$ weak expression rates of Trop-2. MALDI-MS imaging confirmed the presence of Trop- 2 protein in $80 \%$ of the tested tumor samples. This is the first study to demonstrate that several types of SGC express Trop-2 with variable intensity. Since there are currently few systemic treatment options for advanced SGCs, Trop-2 represents a promising target for further clinical studies, for instance, with sacituzumab govitecan.
\end{abstract}

Keywords Salivary gland neoplasms $\cdot$ Immunohistochemistry $\cdot$ Molecular targeted therapy $\cdot$ Retrospective studies · Immunoconjugates $\cdot$ Spectrometry $\cdot$ Mass $\cdot$ Matrix-assisted laser desorption/ionization

\section{Introduction}

Salivary gland carcinomas (SGC) consist of a heterogeneous group of more than a dozen tumors. Although each entity displays recurrent genomic alterations, significant heterogeneity can be found within each group [1,2]. The majority of early

The authors Philipp Wolber and Lisa Nachtsheim have contributed equally to this work.

All authors contributed substantially to the paper in conception, design and writing. This material has never been published and is not currently under evaluation in any other peer-reviewed publication.

Philipp Wolber

Philipp.Wolber@uk-koeln.de

Extended author information available on the last page of the article stage tumors (T1-2) can be treated by tumor resection. Additionally, performance of a neck dissection is recommended in high grade carcinomas even in the absence of clinical or radiological evidence of locoregional metastatic nodal spread and in low-grade tumors with suspicion of (loco-regional) nodal involvement [3]. Generally, in advanced-stage tumors (T3-4), high-grade tumors, positive or close margins or in the presence of lymph node metastasis, vascular and perineural invasion, adjuvant radiation therapy is indicated $[4,5]$. For adenoid cystic carcinoma and other high-risk pathologies (e.g., high-grade mucoepidermoid carcinoma, salivary duct carcinoma), especially those arising in anatomic sites not amenable to wide resection, such as the nasal cavity and paranasal sinuses, many authors recommend adjuvant radiation therapy to increase local control rates even after complete resection due to the tumors' aggressive and often perineural growth $[4,6-10]$. However, the role of adjuvant radiation therapy in 
early-stage tumors without adverse features such as positive margins, perineural invasion and negative neck nodes is contentious, as some authors failed to confirm adjuvant radiation therapy as a prognostic factor $[11,12]$.

Definitive treatment regimens without surgery consisting of combined chemoradiotherapy with curative intent only come into consideration for patients unable to undergo surgery or for unresectable cancers, such as with skull base involvement $[13,14]$. In rare cases, chemotherapy alone can be applied in a neoadjuvant setting to reduce tumor volume for subsequent salvage surgery, but does not play a role in the current standard curative treatment of primary SGC [15-17]. In case of unresectable, recurrent or metastatic cancer, mainly platinumbased palliative chemotherapy regimens are applied. However, randomized controlled trials are lacking and toxicity is considerable [18]. Currently, more information is gathered about possible molecular targets for a tailored therapy for SGCs [19, 20]. Promising results have been achieved with, for example, Herceptin and NTRK inhibitors in selected patients with primarily unresectable disease [10, 21, 22].

Another interesting target is trophoblast cell surface antigen 2 (Trop-2) which is a transmembrane glycoprotein that is involved in a variety of cell signaling pathways including proliferation, survival, self-renewal, and invasion. Cell signaling mediated by Trop-2 involves the expression of Ki-67, activation of MAPK signaling and cell cycle progression by increasing levels of cyclin D1. Trop-2 expression can induce transcription factor AP-1 leading to upregulation of carcinogenesis associated genes [17, 23]. Expression of Trop-2 is associated with poor outcome, particularly in solid tumors [24]. The potential of Trop- 2 as a target for combination therapy using the antibody-drug conjugate sacituzumab govitecan, has already been demonstrated in a number of different tumor entities, such as urothelial carcinoma, squamous cell lung carcinoma and breast cancer [23, 25, 26]. In metastatic triple-negative breast cancer, the FDA has already approved this antibody-drug conjugate [26]. Studies indicate that sacituzumab govitecan mediates anti-tumor responses with varying expression of the Trop- 2 protein $[27,28]$. Until now, the relevance and distribution of Trop-2 protein expression in SGC has not been addressed (Table 1).

The aim of the current study was to test for the extent, distribution and intensity of Trop-2 protein expression in different entities of SGCs using a bimodal approach of immunohistochemistry and matrix-assisted laser desorption/ionization mass spectrometry (MALDI-MS) imaging.

\section{Materials and Methods}

\section{Patients}

We searched the medical records of all patients with SGCs that underwent primary surgery at the Department of Otorhinolaryngology, Head and Neck Surgery, University Hospital of Cologne, Germany between 1990 and 2014, including all patients with sufficient formalin-fixed paraffin-embedded (FFPE) material for further analysis. The study was performed according to the regulations of the Ethics Committee of the University of Cologne.

\section{Data Collection}

Patients' clinical records and histopathologic reports were reviewed with respect to tumor characteristics (stage of disease at the time of diagnosis according to AJCC TNM staging system (8th edition, 2020)) and patient characteristics.

\section{Immunohistochemistry for Trop-2}

All histological diagnoses were reviewed by two pathologists with special expertise in the field (C.A. and A.Q). For tissue microarrays (TMAs), a self-constructed semi-automated precision instrument was used to punch out four tissue cylinders with a diameter of $1.2 \mathrm{~mm}$ from selected tumor tissue blocks. Subsequently, the cylinders were embedded in empty recipient paraffin blocks. From the resulting TMA blocks, sections of $4 \mu \mathrm{m}$ were cut and transferred to an adhesive-coated slide system (Instrumedics Inc., Hackensack, NJ, USA). Immunohistochemistry was performed using a Leica BOND-MAX stainer (Leica Biosystems, Germany), according to the protocol. The anti-Trop2 rabbit IgG monoclonal antibody EPR20043 (Abcam, Cambridge, United Kingdom; dilution 1:1000) was used. After conjugation with an antibody-bound horseradish peroxidase, detection was carried out using Polymer Refine Detection Kit (Leica Biosystems, Germany). Counterstaining was done with hematoxylin and bluing reagent. As the cases in our cohort reflected the entire spectrum of membranous Trop-2 positivity, no reference specimens were used for immunoscore calibration. Several internationally established immunoscores take into account both the intensity of staining and the number of stained tumor cells. One of the more frequently used scores, the $\mathrm{H}$-score, produces equivalent results as the 
semi-quantitative score that was used here [29]. Precisely, membranous basolateral or circumferential Trop-2 staining was assessed as follows: negative ( $<1 \%$ Trop-2 expression of any intensity), weak (weak Trop- 2 protein expression $\leq 70 \%$ or moderate protein expression $\leq 30 \%$ ), moderate (weak Trop-2 protein expression $>70 \%$ or moderate expression $30-70 \%$ or strong expression $\leq 30 \%$ ) or high (moderate Trop-2 protein expression $>70 \%$ or strong expression $>30 \%$ ). In order to analyze a possible intra-tumoral heterogeneity of Trop-2 expression, 20 full sections were additionally stained (five negative cases and two, eight and five cases with weak, moderate and high expression, respectively). The scoring was independently performed by two pathologists with special interest in the field of salivary gland carcinomas (C.A. and A.Q.). In the rare case of a discrepant scoring result, a consensus score was agreed upon.

\section{MALDI-MS Imaging}

For MALDI-MS Imaging, analysis sections consecutive to immunohistochemistry-stained sections were used. Three exemplary cases with high expression, two with moderate, three with weak and two with negative expression of Trop-2 in IHC were analyzed. Sample preparation including deparaffinization, antigen retrieval, tryptic digestion and matrix application was performed according to a previously published study [30]. MALDI-MS Imaging measurements were performed on ultrafleXtreme mass spectrometer (Bruker Daltonik GmbH, Germany). Data acquisition was operated in reflective negative

Table 1 Overview of all included samples organized by histologic group

\begin{tabular}{|c|c|c|c|c|c|c|c|c|c|}
\hline & All $(n=114)$ & $\begin{array}{l}\text { AdCy } \\
(n=22)\end{array}$ & $\begin{array}{l}\text { MuEp } \\
(n=24)\end{array}$ & $\begin{array}{l}\mathrm{ACC} \\
(\mathrm{n}=10)\end{array}$ & $\begin{array}{l}\text { ANOS } \\
(\mathrm{n}=9)\end{array}$ & $\begin{array}{l}\mathrm{SaDu} \\
(\mathrm{n}=23)\end{array}$ & $\begin{array}{l}\text { EpMy } \\
(\mathrm{n}=7)\end{array}$ & $\operatorname{SecC}(n=7)$ & OTH $(\mathrm{n}=12)$ \\
\hline \multicolumn{10}{|l|}{$\begin{array}{l}\text { Localization } \\
\text { (gland) }\end{array}$} \\
\hline Parotid & $103(90.4)$ & $16(72.7)$ & $24(100)$ & $10(100)$ & $9(100)$ & $20(87)$ & $7(100)$ & $6(85.7)$ & $11(91.7)$ \\
\hline $\begin{array}{l}\text { Subman- } \\
\text { dibular }\end{array}$ & $11(9.6)$ & $6(27.3)$ & 0 & 0 & 0 & $3(13)$ & 0 & $1(14.3)$ & $1(8.3)$ \\
\hline \multicolumn{10}{|l|}{$\begin{array}{l}\text { Demograph- } \\
\text { ics }\end{array}$} \\
\hline Female & $59(51.8)$ & $13(59.1)$ & $18(75)$ & $5(50)$ & $5(55.6)$ & $6(26.1)$ & $2(28.6)$ & $3(42.9)$ & $7(58.3)$ \\
\hline Male & $55(48.2)$ & $9(40.9)$ & $6(25)$ & $5(50)$ & $4(44.4)$ & 17 (73.9) & $5(71.4)$ & $4(57.2)$ & $5(41.7)$ \\
\hline Age & $55.8 \pm 17.9$ & $51.5 \pm 14.25$ & $42.5 \pm 16.9$ & $51.4 \pm 18.6$ & $63.9 \pm 15.6$ & $66 \pm 11.5$ & $61 \pm 18.8$ & $48.6 \pm 20$ & $68 \pm 11.1$ \\
\hline \multicolumn{10}{|l|}{$\begin{array}{l}\text { Clinical } \\
\text { parameter }\end{array}$} \\
\hline T1-2 & $57(50)$ & $10(45.5)$ & $15(60)$ & $5(50)$ & $6(66.7)$ & $7(30.4)$ & $4(57.2)$ & $5(71.4)$ & $5(41.7)$ \\
\hline T3-4 & $55(48.2)$ & $11(50)$ & $8(33.3)$ & $5(50)$ & $3(33.3)$ & $16(69.6)$ & $3(42.8)$ & $2(28.6)$ & $7(58.3)$ \\
\hline N/A & $2(1.8)$ & $1(4.5)$ & $1(6.7)$ & 0 & 0 & 0 & 0 & 0 & 0 \\
\hline $\mathrm{N}+$ & $35(30.7)$ & $7(31.8)$ & $3(12.5)$ & $1(10)$ & $3(33.3)$ & $20(87)$ & 0 & $1(14.3)$ & 0 \\
\hline N/A & $4(3.5)$ & $1(4.5)$ & $1(4.2)$ & $1(10)$ & 0 & 0 & 0 & 0 & $1(8.3)$ \\
\hline Pn1 & $38(33.3)$ & $9(40.9)$ & $2(16.7)$ & $2(20)$ & $4(44.4)$ & $17(73.9)$ & 0 & $1(14.3)$ & $3(25)$ \\
\hline N/A & $11(9.6)$ & $4(18.2)$ & $2(16.7)$ & $1(10)$ & 0 & $2(8.7)$ & $1(14.3)$ & 0 & $1(8.3)$ \\
\hline V1 & $9(7.9)$ & 0 & $2(8.3)$ & 0 & $3(33.3)$ & $2(8.7)$ & 0 & 0 & $2(16.7)$ \\
\hline N/A & $11(9.6)$ & $6(27.3)$ & $1(4.2)$ & $1(10)$ & 0 & $2(8.7)$ & 0 & $1(14.3)$ & 0 \\
\hline L1 & 13 (11.4) & 0 & $1(4.2)$ & $1(10)$ & $1(1.1)$ & $9(39.1)$ & 0 & 0 & $1(8.3)$ \\
\hline N/A & $10(8.8)$ & $6(27.3)$ & $1(4.2)$ & $1(10)$ & 0 & $2(8.7)$ & 0 & 0 & 0 \\
\hline \multicolumn{10}{|l|}{$\begin{array}{l}\text { Trop-2 } \\
\text { expression }\end{array}$} \\
\hline Negative & $10(8.8)$ & $1(4.5)$ & 0 & 0 & 0 & $1(4.3)$ & $1(14.3)$ & 0 & $7(58.3)$ \\
\hline Low & $11(9.6)$ & 3 (13.6) & 0 & $2(20)$ & $3(33.3)$ & 0 & $1(14.3)$ & 0 & $2(16.7)$ \\
\hline Moderate & $43(37.7)$ & $12(54.5)$ & 9 & $5(50)$ & $1(11.1)$ & $8(34.8)$ & $3(42.9)$ & $4(57.2)$ & $1(8.3)$ \\
\hline High & $50(43.9)$ & $6(27.4)$ & 15 & $3(30)$ & $5(55.6)$ & $14(60.9)$ & $2(28.6)$ & $3(42.9)$ & $2(16.7)$ \\
\hline
\end{tabular}

Percentages per entity are given in brackets, means are reported \pm standard deviation for metric data. AdCy adenoid cystic carcinoma, $M u E p$ mucoepidermoid carcinoma, ACC acinic cell carcinoma, ANOS adenocarcinoma not otherwise specified; SaDu salivary duct carcinoma, EpMy epithelial-myoepithelial carcinoma, $\operatorname{Sec} C$ secretory carcinoma, $O T H$ others, including polymorphic adenocarcinoma $(\mathrm{n}=1)$; basal cell carcinoma $(n=3)$, myoepithelial carcinoma $(n=2)$, carcinoma ex pleomorphic adenoma $(n=1)$, carcinosarcoma $(n=2)$, poorly differentiated carcinoma $(n=1)$, oncocytic cell carcinoma $(n=2)$; N/A: information not available; Pn1: perineural invasion; V1: vascular invasion; L1: lymphatic invasion 


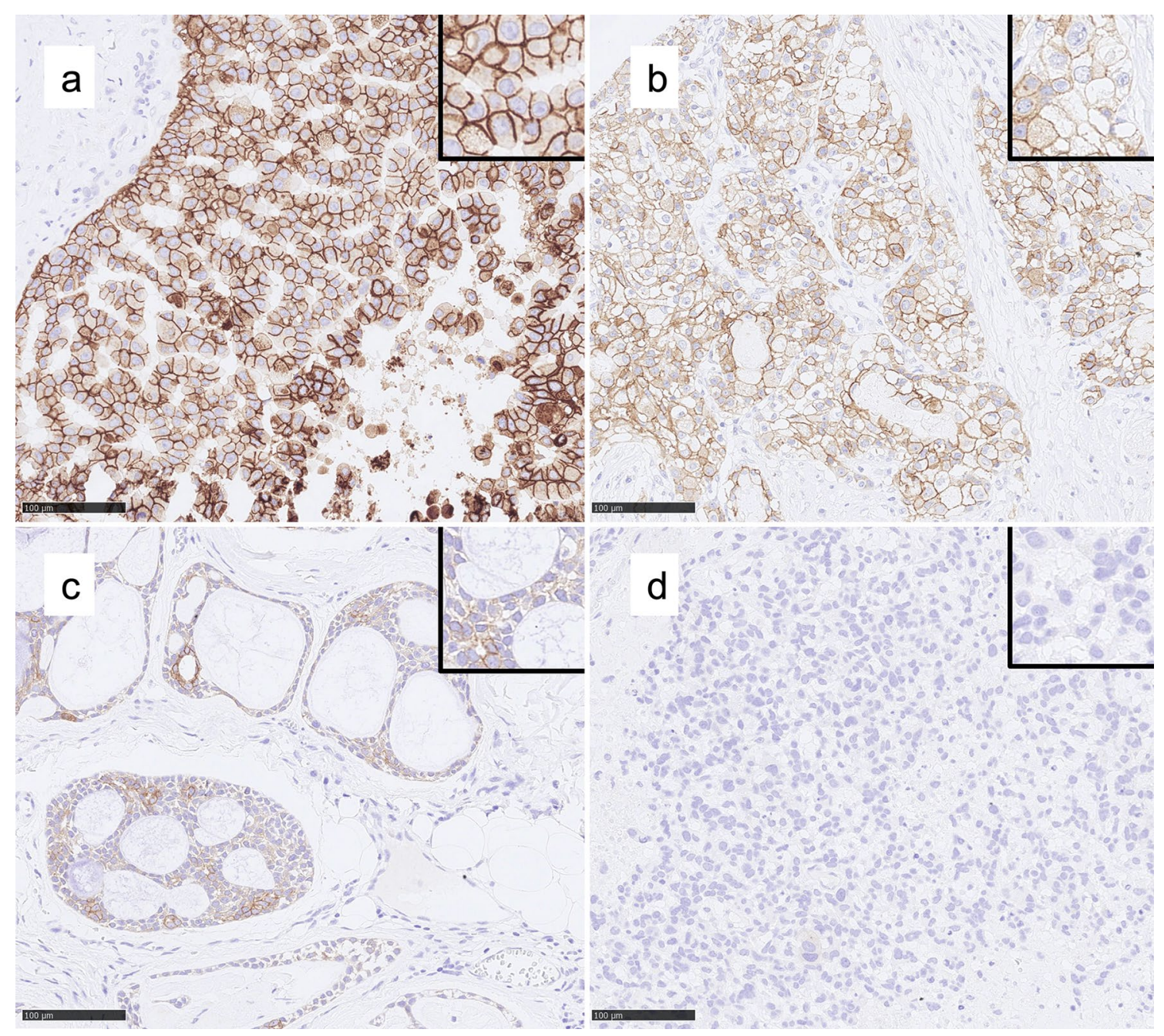

Fig. 1 Exemplary IHC demonstrating membranous Trop-2 expression (magnification 200x, insets 400x). a High expression in a salivary duct carcinoma. b Moderate expression in a salivary duct carcinoma. c Weak expression in an adenoid cystic carcinoma. d Absent expression in a myoepithelial carcinoma

and Fisher's exact test were used to analyze interdependence of Trop-2 expression with clinical parameters. Kaplan-Meier method with $95 \%$ confidence intervals was used to test for disease free survival (DFS) probability rates. For this, statistical significance was tested by using the log-rank test. A p value $<0.05$ was considered statistically significant.

\section{Results}

\section{Demographics}

We identified 114 patients, 59 females (51.8\%) and 55 males (48.2\%). The mean age was $55.8 \pm 17.9$ years (min. 16, max. 86 years) (Table). 


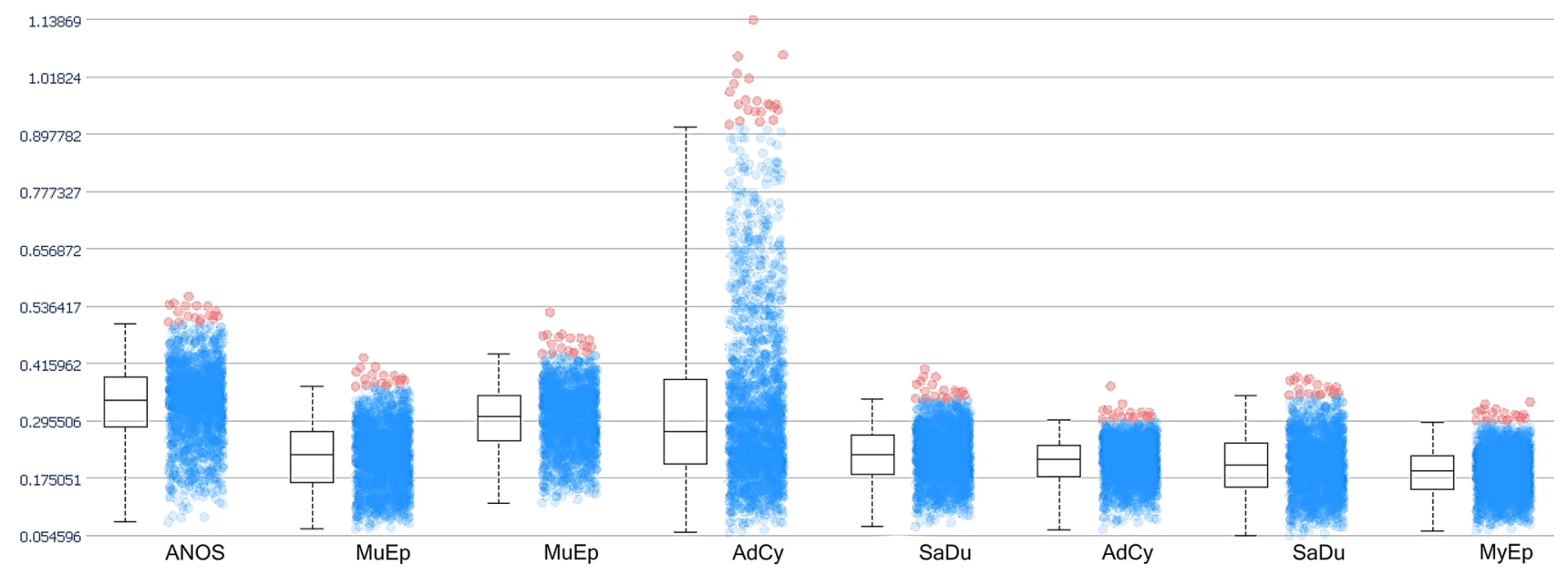

Fig. 2 Trop-2 expression in MALDI-MS imaging. Box plot comparing the mean intensity of the $\mathrm{m} / \mathrm{z}$ value of all detected Trop-2 peptide masses in TMA samples. ANOS adenocarcinoma not-otherwise-spec- ified, $M u E p$ mucoepidermoid carcinoma, $A d C y$ adenoid cystic carcinoma, $S a D u$ salivary duct carcinoma, $M y E p$ myoepithelial carcinoma

out of 24), adenocarcinomas NOS (55.6\%, 5 out of 9) and salivary duct carcinomas $(60.9 \%, 14$ out of 23$)$. All carcinosarcomas $(n=2)$, myoepithelial carcinomas $(n=2)$, undifferentiated carcinomas $(n=1)$ and oncocytic cell carcinomas $(\mathrm{n}=2)$ showed no Trop-2 expression.

The intra-tumoral range of heterogeneity of Trop-2 expression exceeded one in only $10.5 \%$ of cases with high concordance among the different entities, indicating a high homogeneity of Trop- 2 expression. Moreover, 20 full sections were stained with Trop-2 (five with negative, two with weak, eight with moderate and five with high expression in the TMA sections). We observed that all five tumors that were assigned "high" displayed a strong staining in $90 \%$ of the tumor cells. Full sections from tumors with weak or moderate Trop-2 expression confirmed the initial classification with a homogenous expression pattern. Three well to moderately differentiated carcinomas that were assigned "negative" remained completely negative on full sections. Conversely, an undifferentiated carcinoma that has been classified as "negative" revealed a weak staining pattern in a third of the tumor surface. Another initially "negative" tumor, a de-differentiated salivary duct carcinoma was completely negative in the poorly differentiated component but highly positive for Trop-2 ("high") in the well-differentiated part.

No statistically significant difference in Trop- 2 expression was found between the most prevalent SGC entities (adenoid cystic carcinoma, mucoepidermoid carcinoma, acinic cell carcinoma, adenocarcinoma NOS, salivary duct carcinoma, epithelial myoepithelial carcinoma and secretory carcinoma; $\mathrm{p}=0.462$ ). Moreover, Trop- 2 expression showed no correlation with tumor localization (parotid gland vs. submandibular gland; $p=0.32)$, sex $(p=0.744)$, presence of nodal 
metastases $(p=0.721)$, perineural invasion $(p=0.707)$, vascular invasion $(\mathrm{p}=0.576)$, lymphatic invasion $(\mathrm{p}=1.000)$. Regarding T-stage, there was a trend towards smaller tumors showing Trop-2 expression $(\mathrm{p}=0.051)$.

For validation of the immunohistochemistry data, MALDI-MS imaging was performed on 10 exemplary samples. Out of the putative tryptic peptides from Trop-2, seven could be detected. Trop- 2 protein was detected in $80 \%$ of the samples (Fig. 2). One of the Trop-2 positive samples showed no expression of Trop-2 protein in IHC. The highest intensities of Trop-2 expression in MALDIMS imaging were in one adenoid cystic carcinoma, one mucoepidermoid carcinoma and one adenocarcinoma NOS. Out of these samples, two showed weak and one strong Trop-2 expression in IHC. Out of the two samples with no Trop-2 expression in MALDI-MS imaging, one mucoepidermoid carcinoma showed moderate expression and one adenoid cystic carcinoma showed no expression in IHC.

\section{Survival}

After a median follow-up of 52 months, the total 5-year disease free survival (DFS) rate was $76.1 \%$ (86 out of 114). The lowest 5-year DFS rate was found for adenocarcinoma NOS (50\%) and the highest for secretory carcinoma (100\%) and acinic cell carcinoma (90\%). Trop-2 expression showed no correlation with 5-year DFS (log rank test, $\mathrm{p}=0.225)$. 5-year DFS rate was significantly better for patients in T-stage 1 and 2 (89.3\%) compared to T-stage 3 and 4 (61.8\%; log rank test, $\mathrm{p}=0.001)$. Lymphatic invasion was found to be negatively correlated with DFS ( $83.3 \%$ for L0 vs. $46.2 \%$ for L1; $\mathrm{p}=0.001)$. No correlation was found for $\operatorname{sex}(\mathrm{p}=0.712)$.

\section{Discussion}

This is the first study to determine the protein expression of Trop-2 using different techniques in salivary gland carcinomas. Trop- 2 is a transmembrane protein with an extracellular domain and its expression is upregulated in tumors cells relative to normal cells [23]. It is involved in several molecular pathways associated with cancer development and has multiple binding partners. Trop-2 expression can increase the expression of $\mathrm{Ki}-67$ causing $\mathrm{Ca} 2+$ mobilization from internal stores.

MAPK signaling is activated by Trop-2 leading to increased levels of phosphorylated ERK1 and ERK2 [17]. Activation of ERK can putatively induce proteasomal degradation of FOXO3a and thereby inhibit cell death [31]. Also, MAPK signaling and cell cycle progression are further stimulated by $\mathrm{Ca} 2+$. Trop- 2 expression can increase levels of cyclin D1 and cyclin E, helping to mediate ERK1/2 cell cycle progression. Subsequently, ERK signaling induces AP-1 transcription factor which plays a crucial role in the regulation of tumor-associated target genes during carcinogenesis [32]. AP-1 can induce angiogenesis via VEGF (vascular endothelial growth factor), activate cell proliferation via cyclins and CDKs and cause cell invasion and metastasis via matrix metalloproteinases, podoplanin, Ezrin and CD44. Furthermore, AP-1 can induce epithelial to mesenchymal transition via podoplanin allowing for the nuclear translocation of $\beta$-catenin, eventually causing cell growth via downstream effectors [17].

Trop-2 overexpression has been described in a variety of malignant tumors, such as in colorectal, esophageal, lung, ovarian or head and neck cancer [17]. Several clinical trials have been investigating Trop- 2 as a potential target. PF-06664178, an antibody-drug conjugate delivering an auristatin microtubule inhibitor, was tested in a phase I study in patients with advanced therapy-resistant solid tumors. Eleven out of 31 patients showed stable disease and no complete or partial response was observed [33]. DS-1062a, an antibody-drug conjugate delivering a topoisomerase I inhibitor and derivate of exatecan, is currently being tested for the treatment of unresectable advanced and incurable NSCLC in an ongoing first in human study [23]. Sacituzumab govitecan (or IMMU-132), an antibody-drug conjugate comprising the active metabolite of irinotecan (SN-38) conjugated to an anti-Trop-2 antibody, has been investigated in several trials. In a phase I study in patients with platinum-resistant urothelial carcinoma, it was well tolerated and three out of six patients had a clinically significant response, with a progression free survival of 6.7-8.2 months [34]. Due to these results, a phase II trial has been initiated. For metastatic NSCLC patients, Sacituzumab govitecan was tested in a phase II study with 54 pretreated patients. A clinical benefit rate, defined as complete or partial response or stable disease for more than four months was observed in $43 \%$ of the patients. The median response duration to therapy was six months, with a median overall survival of 9.5 months and median progression free survival of 5.2 months [35]. For SCLC patients, Sacituzumab govitecan has also been evaluated in a phase II study. In $60 \%$ of the patients tumor shrinkage was observed and a clinical benefit rate was reported for $34 \%$ with a median overall survival of 7.5 months and progression free survival of 3.7 months [25]. However, the most promising results have been achieved for triple-negative breast cancer. Bardia et al. have recently investigated the use of sacituzumab govitecan in 108 cases of refractory triple-negative breast cancer. They reported a response rate of $33.3 \%$, including a complete response in $2.8 \%$, with a median duration of response of 7.7 months and a median progression free survival of 5.5 months [26]. These results have led to the approval of sacituzumab govitecan (TRODELVY, Immunomedics, Inc.) by the U.S. Food 
and Drug Administration for adult patients with metastatic triple-negative breast cancer who received at least two prior therapies for metastatic disease.

In the current study, among the $91.2 \%$ of SGCs with Trop- 2 expression, $81.6 \%$ exhibited a high to moderate protein concentration in immunohistochemistry. More than half of the cohort consisted of adenoid cystic, mucoepidermoid and salivary duct carcinomas. Thus, the most prevalent tumor entities are well represented in the collective. The rates of Trop- 2 expression were particularly high in adenoid cystic carcinomas, salivary duct carcinomas, epithelial myoepithelial carcinomas and mucoepidermoid carcinomas. The results of IHC were validated with MALDI-MS Imaging on 10 exemplary samples. Trop-2 protein expression was detected in $80 \%$. There were two discordant findings regarding Trop-2 expression. One sample showed negative Trop-2 expression in IHC but protein expression in MALDI-MS imaging, and another with negative Trop- 2 expression in MALDI-MS imaging showed moderate expression in IHC. A possible explanation for this could be the fact that consecutive TMA sections were used. We have also considered the heterogeneity of protein expression within the tumor. For this purpose, we analyzed all tumors with four tumor biopsies from different tumor sites in TMA format and additionally 20 tumors on full tumor sections. Most of the tumors (89.5\%) showed a homogeneous expression of Trop2. Carcinomas with a high Trop-2 pattern were homogeneously positive even on full section, while undifferentiated or dedifferentiated carcinomas displayed a more heterogeneous Trop-2 expression. This underlines the significance of our TMA-based analysis and allows conclusions to be drawn about the potential therapeutic effectiveness of sacituzumab govitecan in SGCs. The more homogeneously a therapyrelevant biomarker is expressed in the tumor, the more likely it is to be effectively targeted.

In comparably designed studies (e.g., Gatsby study), in which a biomarker (Her2/neu) - also determined on the tumor cells-was combined with an antibody-drug conjugate (trastuzumab emtansine) specifically directed against the biomarker, the very heterogeneous distribution of Her2/ neu in gastric cancer was a possible relevant reason for the failure of this study [36]. The same drug combination is effective in breast cancer, possibly because (in contrast to gastric cancer) breast cancer usually shows a very homogeneous expression of Her2/neu.

Given the homogeneous expression of Trop-2 in SGC, future clinical studies will have to verify the relationship between Trop- 2 protein expression and sacituzumab govitecan. From today's perspective, however, SGCs seem to be good candidates for a Trop-2-targeted therapy.

In our cohort, Trop-2 expression showed no correlation with the presence of nodal metastases, perineural, vascular or lymphatic invasion and 5-year disease free survival. This is in contrast to the findings of a meta-analysis by Ping et al., who reported a significant association of Trop-2 overexpression and short disease free survival in different solid tumors [24]. Therefore, the role of Trop-2 as a prognostic biomarker in SGCs remains inconclusive.

Limitations of our study could be the retrospective fashion of our analyses. The limited number of cases among each individual tumor type make it difficult to establish correlations with clinical parameters or outcome. However, due to the low incidence of certain SGC subtypes, desirable prospective studies with sufficient participants are particularly challenging. Nevertheless, despite these shortcomings we are convinced that the results of this exploratory study can be followed up in clinical studies with Trop- 2 as a target in salivary gland carcinomas.

In summary, we were able to demonstrate that several types of salivary gland carcinoma immunohistochemically express Trop- 2 with variable intensity. Since there are currently few systemic treatment options for advanced SGCs, Trop-2 represents a promising target for further clinical studies, such as with sacituzumab govitecan.

Funding Open Access funding enabled and organized by Projekt DEAL. This research did not receive any specific grant from funding agencies in the public, commercial, or not-for-profit sectors. No funding was obtained.

\section{Compliance with Ethical Standards}

Conflict of interest The authors declare no conflict of interest.

Ethical Approval This research study was conducted retrospectively from data obtained for clinical purposes. We consulted with the IRB of the University of Cologne who determined that our study did not need ethical approval.

Open Access This article is licensed under a Creative Commons Attribution 4.0 International License, which permits use, sharing, adaptation, distribution and reproduction in any medium or format, as long as you give appropriate credit to the original author(s) and the source, provide a link to the Creative Commons licence, and indicate if changes were made. The images or other third party material in this article are included in the article's Creative Commons licence, unless indicated otherwise in a credit line to the material. If material is not included in the article's Creative Commons licence and your intended use is not permitted by statutory regulation or exceeds the permitted use, you will need to obtain permission directly from the copyright holder. To view a copy of this licence, visit http://creativecommons.org/licenses/by/4.0/.

\section{References}

1. Meinrath J, Haak A, Igci N, Dalvi P, Arolt C, Meemboor S, et al. Expression profiling on subclasses of primary parotid gland carcinomas. Oncotarget. 2020;11:4123-37. 
2. Seethala RR. Salivary gland tumors: current concepts and controversies. Surg Pathol Clin. 2017;10:155-76.

3. Westergaard-Nielsen M, Rosenberg T, Gerke O, Dyrvig A-K, Godballe C, Bjørndal K. Elective neck dissection in patients with salivary gland carcinoma: a systematic review and meta-analysis. J Oral Pathol Med Off Publ Int Assoc Oral Pathol Am Acad Oral Pathol. 2020;49:606-16.

4. Terhaard CHJ. Postoperative and primary radiotherapy for salivary gland carcinomas: indications, techniques, and results. Int J Radiat Oncol Biol Phys. 2007;69:S52-55.

5. Lee A, Givi B, Osborn VW, Schwartz D, Schreiber D. Patterns of care and survival of adjuvant radiation for major salivary adenoid cystic carcinoma. Laryngoscope. 2017;127:2057-62.

6. Hu W, Hu J, Huang Q, Gao J, Yang J, Qiu X, et al. Particle beam radiation therapy for adenoid cystic carcinoma of the nasal cavity and paranasal sinuses. Front Oncol. 2020;10:572493.

7. Sood S, McGurk M, Vaz F. Management of Salivary Gland Tumours: United Kingdom National Multidisciplinary Guidelines. J Laryngol Otol. 2016;130:S142-9.

8. Ikawa H, Koto M, Takagi R, Ebner DK, Hasegawa A, Naganawa $\mathrm{K}$, et al. Prognostic factors of adenoid cystic carcinoma of the head and neck in carbon-ion radiotherapy: The impact of histological subtypes. Radiother Oncol J Eur Soc Ther Radiol Oncol. 2017;123:387-93.

9. Takebayashi S, Shinohara S, Tamaki H, Tateya I, Kitamura M, Mizuta M, et al. Adenoid cystic carcinoma of the head and neck: a retrospective multicenter study. Acta Otolaryngol (Stockh). 2018;138:73-9.

10. Wang X, Luo Y, Li M, Yan H, Sun M, Fan T. Management of salivary gland carcinomas - a review. Oncotarget. 2017;8:3946-56.

11. Meyers M, Granger B, Herman P, Janot F, Garrel R, Fakhry N, et al. Head and neck adenoid cystic carcinoma: A prospective multicenter REFCOR study of 95 cases. Eur Ann Otorhinolaryngol Head Neck Dis. 2016;133:13-7.

12. Oplatek A, Ozer E, Agrawal A, Bapna S, Schuller DE. Patterns of recurrence and survival of head and neck adenoid cystic carcinoma after definitive resection. Laryngoscope. 2010;120:65-70.

13. Chen AM, Bucci MK, Quivey JM, Garcia J, Eisele DW, Fu KK. Long-term outcome of patients treated by radiation therapy alone for salivary gland carcinomas. Int J Radiat Oncol Biol Phys. 2006;66:1044-50.

14. Hsieh C-E, Lin C-Y, Lee L-Y, Yang L-Y, Wang C-C, Wang H-M, et al. Adding concurrent chemotherapy to postoperative radiotherapy improves locoregional control but Not overall survival in patients with salivary gland adenoid cystic carcinoma-a propensity score matched study. Radiat Oncol Lond Engl. 2016;11:47.

15. Mousa A, Rahimi K, Warkus T. Neoadjuvant chemoradiotherapy followed by radical vulvectomy for adenoid cystic carcinoma of Bartholin's gland: a case report and review of the literature. Eur J Gynaecol Oncol. 2016;37:113-6.

16. Jang SY, Kim DJ, Kim CY, Wu CZ, Yoon JS, Lee SY. Neoadjuvant intra-arterial chemotherapy in patients with primary lacrimal adenoid cystic carcinoma. Cancer Imaging Off Publ Int Cancer Imaging Soc. 2014;14:19.

17. Shvartsur A, Bonavida B. Trop2 and its overexpression in cancers: regulation and clinical/therapeutic implications. Genes Cancer. 2015;6:84-105.

18. Alfieri S, Granata R, Bergamini C, Resteghini C, Bossi P, Licitra LF, et al. Systemic therapy in metastatic salivary gland carcinomas: A pathology-driven paradigm? Oral Oncol. 2017;66:58-63.

19. Seethala RR, Stenman G. Update from the 4th Edition of the World Health Organization Classification of Head and Neck Tumours: Tumors of the Salivary Gland. Head Neck Pathol. 2017;11:55-67.
20. Schmitt NC, Kang H, Sharma A. Salivary duct carcinoma: An aggressive salivary gland malignancy with opportunities for targeted therapy. Oral Oncol. 2017;74:40-8.

21. Hong DS, DuBois SG, Kummar S, Farago AF, Albert CM, Rohrberg KS, et al. Larotrectinib in patients with TRK fusion-positive solid tumours: a pooled analysis of three phase $1 / 2$ clinical trials. Lancet Oncol. 2020;21:531-40.

22. Gibo T, Sekiguchi N, Gomi D, Noguchi T, Fukushima T, Kobayashi $\mathrm{T}$, et al. Targeted therapy with trastuzumab for epidermal growth factor receptor 2 (HER2)-positive advanced salivary duct carcinoma: A case report. Mol Clin Oncol. 2019;11:111-5.

23. Zaman S, Jadid H, Denson AC, Gray JE. Targeting Trop-2 in solid tumors: future prospects. OncoTargets Ther. 2019;12:1781-90.

24. Zeng P, Chen M-B, Zhou L-N, Tang M, Liu C-Y, Lu P-H. Impact of TROP2 expression on prognosis in solid tumors: A Systematic Review and Meta-analysis. Sci Rep. 2016;6:33658.

25. Gray JE, Heist RS, Starodub AN, Camidge DR, Kio EA, Masters GA, et al. Therapy of Small Cell Lung Cancer (SCLC) with a Topoisomerase-I-inhibiting Antibody-Drug Conjugate (ADC) Targeting Trop-2, Sacituzumab Govitecan. Clin Cancer Res Off J Am Assoc Cancer Res. 2017;23:5711-9.

26. Bardia A, Mayer IA, Vahdat LT, Tolaney SM, Isakoff SJ, Diamond JR, et al. Sacituzumab govitecan-hziy in refractory metastatic triple-negative breast cancer. N Engl J Med. 2019;380:741-51.

27. Cardillo TM, Govindan SV, Sharkey RM, Trisal P, Arrojo R, Liu D, et al. Sacituzumab govitecan (IMMU-132), an Anti-Trop-2/ SN-38 antibody-drug conjugate: characterization and efficacy in pancreatic, gastric, and other cancers. Bioconjug Chem. 2015;26:919-31.

28. Goldenberg DM, Cardillo TM, Govindan SV, Rossi EA, Sharkey RM. Trop-2 is a novel target for solid cancer therapy with sacituzumab govitecan (IMMU-132), an antibody-drug conjugate (ADC). Oncotarget. 2015;6:22496-512.

29. Moentenich V, Comut E, Gebauer F, Tuchscherer A, Bruns C, Schroeder W, et al. Mesothelin expression in esophageal adenocarcinoma and squamous cell carcinoma and its possible impact on future treatment strategies. Ther Adv Med Oncol. 2020;12:1758835920917571.

30. Arolt C, Meyer M, Hoffmann F, Wagener-Ryczek S, Schwarz D, Nachtsheim L, et al. Expression Profiling of Extracellular Matrix Genes Reveals Global and Entity-Specific Characteristics in Adenoid Cystic, Mucoepidermoid and Salivary Duct Carcinomas. Cancers. 2020;12.

31. Koo C-Y, Muir KW. Lam EW-F. FOXM1: From cancer initiation to progression and treatment. Biochim Biophys Acta. 2012;1819:28-37.

32. Cubas R, Zhang S, Li M, Chen C, Yao Q. Trop2 expression contributes to tumor pathogenesis by activating the ERK MAPK pathway. Mol Cancer. 2010;9:253.

33. King GT, Eaton KD, Beagle BR, Zopf CJ, Wong GY, Krupka HI, et al. A phase 1, dose-escalation study of PF-06664178, an anti-Trop-2/Aur0101 antibody-drug conjugate in patients with advanced or metastatic solid tumors. Invest New Drugs. 2018:36:836-47.

34. Faltas B, Goldenberg DM, Ocean AJ, Govindan SV, Wilhelm F, Sharkey RM, et al. Sacituzumab govitecan, a novel antibody-drug conjugate, in patients with metastatic platinum-resistant urothelial carcinoma. Clin Genitourin Cancer. 2016;14:e75-79.

35. Heist RS, Guarino MJ, Masters G, Purcell WT, Starodub AN, Horn L, et al. Therapy of advanced non-small-cell lung cancer with an SN-38-Anti-Trop-2 drug conjugate, sacituzumab govitecan. J Clin Oncol Off J Am Soc Clin Oncol. 2017;35:2790-7.

36. Thuss-Patience PC, Shah MA, Ohtsu A, Van Cutsem E, Ajani JA, Castro $\mathrm{H}$, et al. Trastuzumab emtansine versus taxane use for previously treated HER2-positive locally advanced or metastatic gastric or gastro-oesophageal junction adenocarcinoma 
(GATSBY): an international randomised, open-label, adaptive, phase 2/3 study. Lancet Oncol. 2017;18:640-53.

Publisher's Note Springer Nature remains neutral with regard to jurisdictional claims in published maps and institutional affiliations.

\section{Authors and Affiliations}

\section{Philipp Wolber ${ }^{1}$ (D) Lisa Nachtsheim ${ }^{1}$. Franziska Hoffmann ${ }^{2,3}$. Jens Peter Klußmann ${ }^{1,4}$ • Moritz Meyer ${ }^{5}$.

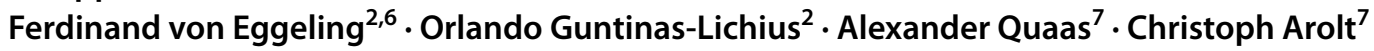

1 Department of Otorhinolaryngology, Head and Neck Surgery, University of Cologne, Medical Faculty, Cologne, Germany

2 Department of Otorhinolaryngology, Head and Neck Surgery, Jena University Hospital, Friedrich-Schiller-University Jena, Jena, Germany

3 Department of Otorhinolaryngology, MALDI Imaging and Innovative Biophotonics, Jena University Hospital, Friedrich-Schiller-University Jena, Jena, Germany

4 Center for Molecular Medicine Cologne (CMMC), University of Cologne, Faculty of Medicine and University Hospital Cologne, Cologne, Germany
5 Department of Otorhinolaryngology, Head and Neck Surgery, University Hospital Essen, University Duisburg-Essen, Essen, Germany

6 DFG Core Unit Jena Biophotonic and Imaging Laboratory (JBIL), MALDI Imaging, Core Unit Proteome Analysis, Jena University Hospital, Friedrich-Schiller-University Jena, Jena, Germany

7 Department of Pathology, University of Cologne, Medical Faculty, Cologne, Germany 